\title{
Proposal for an All-Optical Flip-Flop Using a Single Distributed Feedback Laser Diode
}

\author{
Koen Huybrechts, Student Member, IEEE, Wouter D'Oosterlinck, Student Member, IEEE, \\ Geert Morthier, Senior Member, IEEE, and Roel Baets, Fellow, IEEE
}

\begin{abstract}
We introduce a new concept for an all-optical flip-flop based on a single distributed feedback laser. The injection of external light into the laser results in a nonuniform carrier distribution. We will show numerically that this nonlinear effect of the carriers gives rise to a bistability that can be exploited for fast flip-flop operation.
\end{abstract}

Index Terms-All-optical flip-flops, distributed feedback (DFB) lasers, optical bistability.

\section{INTRODUCTION}

$\mathbf{P}$ ACKET- or burst-switched optical networks are gaining a lot of interest due to the increasing demand for faster network traffic [1]. All-optical flip-flops offer one of the key functionalities in realizing these optical networks by acting as optical memory elements and storing temporal decisions in photonic packet routers. Several designs for all-optical flip-flops based on bistable effects have been proposed so far. The main principle in these designs is the combination of a nonlinear effect and a feedback mechanism thus leading to a bistable device. Many of the devices that have been proposed are based on nonlinear effects in the gain such as coupled laser diodes [2], multimode interference bistable laser diodes [3], and devices based on semiconductor-optical-amplifier-based Mach-Zehnder interferometers with a feedback loop [4]. All these devices are, however, relatively complex.

Several attempts have already been made to use the advantage of the small spectral linewidth offered by distributed feedback (DFB) lasers for all-optical flip-flop operation. In [5], a two-segment multiquantum-well DFB laser is demonstrated for bistable switching: one of the segments is biased below threshold to act as a saturable absorber while the other segment provides the gain. Due to the high carrier lifetimes in the saturable absorber, however, the repetition rate is limited to only $1 \mathrm{GHz}$. Dispersive bistabilities occuring in DFB amplifiers can also be used to achieve flip-flop operation, as shown in [6], [7], but they have no fast switching OFF and require a tight wavelength control.

Manuscript received August 1, 2007; revised September 3, 2007. The work of K. Huybrechts and W. D'Oosterlinck was supported by the Institute for the Promotion of Innovation through Science and Technology in Flanders (IWTVlaanderen) under a specialization grant.

The authors are with the Photonics Research Group, Department of Information Technology (INTEC), Ghent University-IMEC, B-9000 Ghent, Belgium (e-mail: koen.huybrechts@intec.ugent.be; wouter.doosterlinck@intec. ugent.be; geert.morthier@intec.ugent.be; roel.baets@intec.ugent.be).

Digital Object Identifier 10.1109/LPT.2007.911007

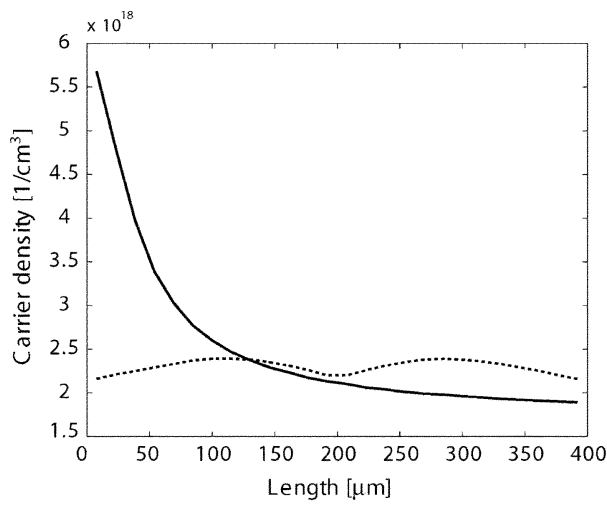

Fig. 1. Longitudinal distribution of the carriers in the DFB laser. Dashed line: lasing state. Solid line: nonlasing state.

Here we propose and numerically analyze a new design for an all-optical flip-flop: a single $\lambda / 4$-shifted DFB laser with antireflection (AR)-coated facets and biased above threshold. By injecting external light with a wavelength different from the lasing wavelength into the DFB laser, a bistability can be obtained. This bistability is observed in the lasing light as well as in the amplification of the external light and is due to nonlinear effects having their origins in the carrier distribution, i.e., spatial hole burning.

\section{OPERATION PRINCIPLE}

When injecting continuous-wave $(\mathrm{CW})$ light into a DFB-laser, two stable states can be possible for the same input power. This bistability arises from the strong influence of the carrier distribution on the threshold characteristics of a DFB laser. In one of the states the laser is lasing and the externally injected light is weakly amplified due to gain clamping. The other state, in contrast, has a very high amplification of the external light, resulting in a strong nonuniform distribution of the carriers. It is well-known that this sort of spatial hole burning effect can increase the threshold of a DFB laser diode, ultimately causing the laser to switch OFF. Therefore, all the injected carriers in the device will be used to amplify the injected light.

The two different states of the carrier density distribution are simulated with a commercial software package [8] and are depicted in Fig. 1 for an AR-coated DFB laser with a $\lambda / 4$-shift, a length of $400 \mu \mathrm{m}$, and a normalized coupling coefficient $\kappa L$ of 1.2. The laser is lasing at a wavelength of $1.57 \mu \mathrm{m}$ and we inject CW light of $1.56 \mu \mathrm{m}$ to simulate the hysteresis curve. In Fig. 2(a), one can see the influence of the injected light on the 


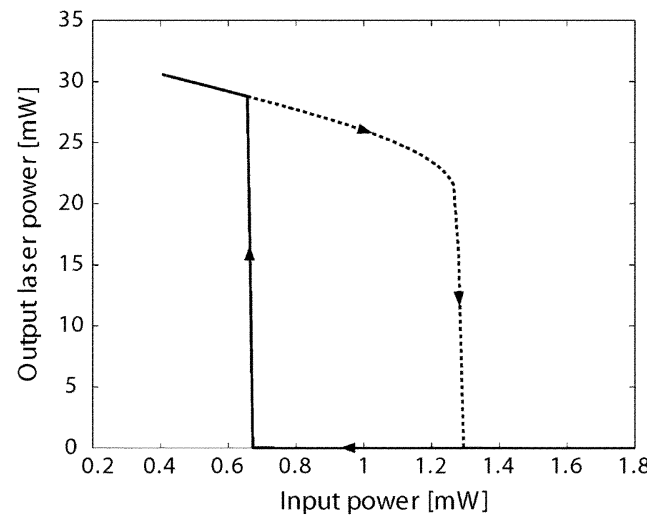

(a)

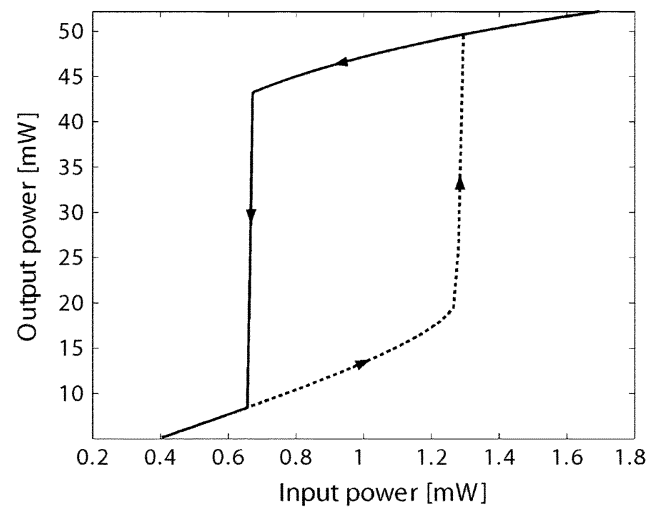

(b)

Fig. 2. Simulation results of the bistable behavior of a $\lambda / 4$-shifted and AR-coated multiquantum-well DFB-laser with length $400 \mu \mathrm{m}, \kappa L 1.2$, and active layer thickness $40 \mathrm{~nm}$. The laser is electrically pumped with $I / I_{\mathrm{th}}=4$ and $I_{\mathrm{th}}=42.5 \mathrm{~mA}$. (a) Laser output power as a function of the power of the injected light. (b) Amplification of the injected light.

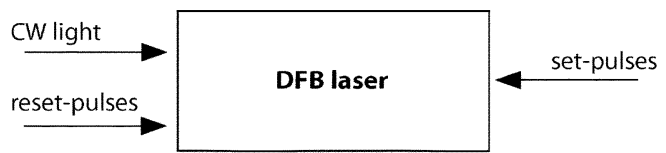

Fig. 3. Schematic representation of the all-optical flip-flop.

lasing power, while in Fig. 2(b), the bistability of the amplification of the injected light is depicted. There are no strict limitations for the wavelength of the injected light, except for the fact that it should not be too close to the lasing wavelength (more than 1-nm difference) to avoid interaction with the DFB grating.

We can exploit this bistability for all-optical flip-flop operation (see Fig. 3). To make the device operative in the bistable regime, a bias CW light is injected in the DFB laser. Switching from the lasing state to the nonlasing state can be done by injecting a short but strong pulse at the same side of the device as the $\mathrm{CW}$ light. This will cause a nonuniform carrier distribution and thus increase the lasing threshold. The uniform carrier distribution can then be restored by injecting a light pulse at the other side of the device. This will reduce the laser threshold and allow the laser field to switch ON again.

\section{STATiC Behaviour}

The bistability curve depends on the parameters of the DFB laser. We have investigated the influence of the bias current, the

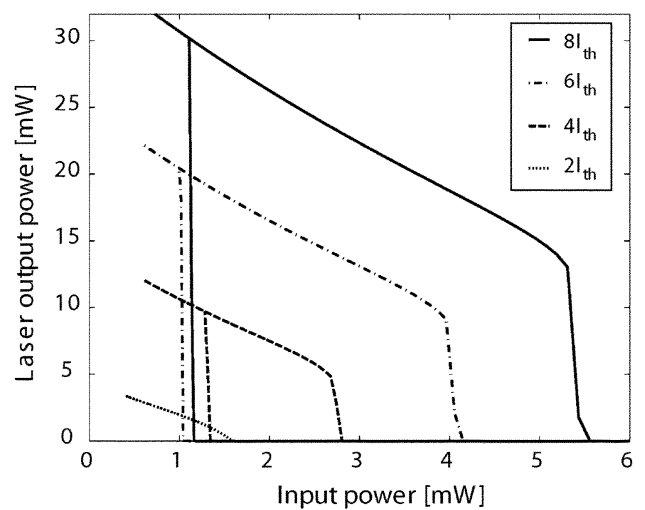

Fig. 4. Influence of the bias current on the hysteresis characteristics for a DFB laser of length $600 \mu \mathrm{m}, \kappa L=1.8$, and $I_{\mathrm{th}}=26.5 \mathrm{~mA}$.

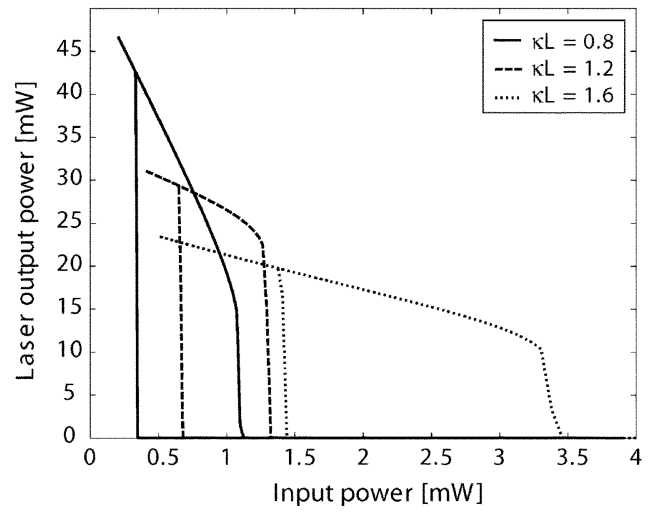

Fig. 5. Influence of the coupling coefficient on the hysteresis characteristics for a DFB laser of length $400 \mu \mathrm{m}$ and $I_{\text {bias }}=4 I_{\mathrm{th}}$.

normalized coupling coefficient $\kappa L$, the length and the losses for AR-coated DFB lasers with a quarter wavelength shift. The AR coatings are necessary to achieve a sufficient nonuniformity of the carriers and the $\lambda / 4$-shift is necessary to increase the influence of spatial hole burning in the laser. Changes in wavelength have almost no influence on the hysteresis characteristics as long as there is a significant difference between the wavelengths of the injected light and the lasing light.

1) Influence of the bias current: The influence of the bias current on the hysteresis curve is depicted in Fig. 4. The bistable domain becomes smaller when decreasing the bias current. The reason is that for low current the laser switches OFF sooner due to overall carrier depletion by the injected field.

2) Influence of the normalized coupling coefficient: As can be seen in Fig. 5, the hysteresis curve will narrow and shift for decreasing $\kappa L$ values. This can be explained by the increase of the threshold gain for decreasing $\kappa L$ value. This results in a higher amplification of the injected light and thus bistable behaviour for a lower injection power.

3) Influence of the fixed internal loss: The influence of the fixed internal loss (including the material and scattering losses) is studied in Fig. 6. We can observe that when the losses are higher, the laser will switch OFF at a slightly lower input power of the $\mathrm{CW}$ light for fixed values of $I_{\text {bias }} / I_{\text {th }}$. 


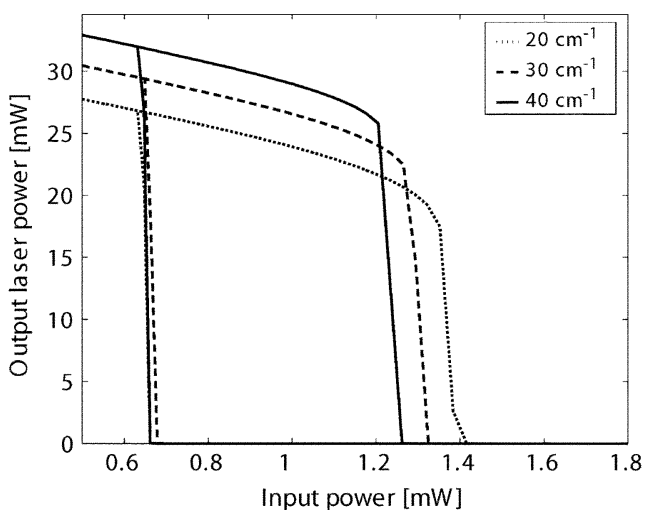

Fig. 6. Influence of the fixed internal loss on the hysteresis characteristics for DFB lasers with $L=400 \mu \mathrm{m}, \kappa L=1.2$, and $I_{\text {bias }}=4 I_{\mathrm{th}}$.

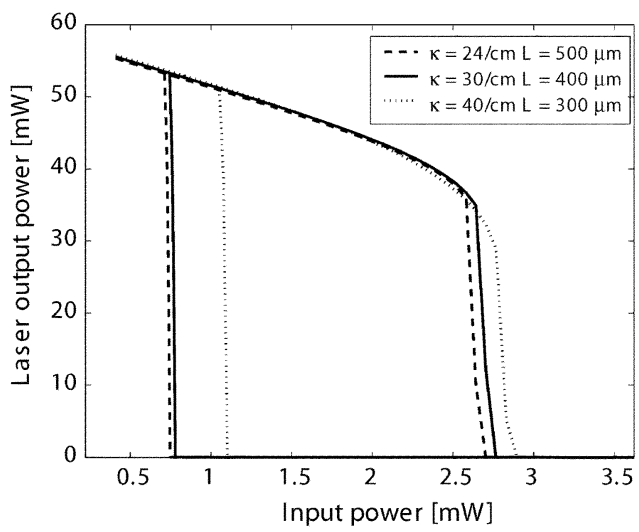

Fig. 7. Influence of the length/coupling coefficient on the hysteresis characteristics for different configurations with $\kappa L=1.2$ with the losses zero and with bias current such that the output powers are equal when no light is injected.

4) Influence of the length and the grating coupling: In Fig. 7, the bistability curves are plotted for different configurations with a fixed normalized coupling coefficient, without loss and with a bias current chosen such that the output powers are equal when no light is injected. The curves seem to overlap quite well and thus we can assume that the parameters described above are the main factors influencing the form of the bistability curve.

\section{DYNAMIC BeHAVIOUR}

The dynamic behaviour of the DFB flip-flop with the specifications given in Fig. 2 is simulated with a CW light injection of $1 \mathrm{~mW}$ at one side of the laser cavity. On the same side as the $\mathrm{CW}$ light, we also inject the reset pulses to move out of the hysteresis curve and switch OFF the laser. The set pulses are injected on the other side of the device to restore the uniformity. The advantage of this approach is that we can use exclusively positive pulses. We simulate this for Gaussian pulses of $200 \mathrm{ps}$ with

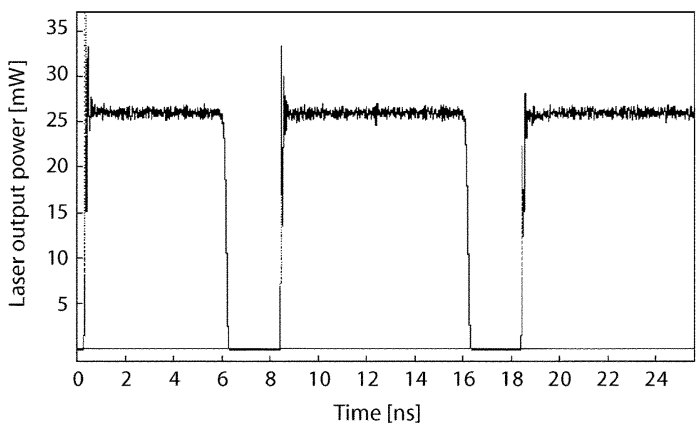

Fig. 8. Illustration of the all-optical flip-flop behaviour in a single DFB-laser with the specifications of Fig. 2.

switch pulse energies of about $500 \mathrm{fJ}$ (see Fig. 8). The contrast ratio is $32 \mathrm{~dB}$ and we obtain switching times of about $250 \mathrm{ps}$. The laser will switch on faster by increasing the pulse energy, but the overshoot will be higher resulting in a longer stabilization time. Repetition rates up to $1.2 \mathrm{GHz}$ can be achieved. There are no strict limitations on the wavelength of the pulses as discussed before.

\section{CONCLUSION}

We demonstrated the use of a single DFB laser diode as an all-optical flip-flop. The shape of the hysteresis curve depends on the parameters of the laser and on the bias current. Dynamic behaviour using positive pulses is simulated and predicts switching times of $250 \mathrm{ps}$.

\section{REFERENCES}

[1] H. Doiren, M. Hill, Y. Liu, N. Calabretta, A. Srivatsa, F. Huijskens, H. de Waardt, and G. Khoe, "Optical packet switching and buffering by using all-optical signal processing methods," J. Lightw. Technol., vol. 21, no. 1, pp. 2-12, Jan. 2003.

[2] M. T. Hill, H. de Waardt, G. Khoe, and H. Dorren, "All-optical flip-flop based on coupled laser diodes," IEEE J. Quantum Electron., vol. 37, no. 3, pp. 405-413, Mar. 2001.

[3] M. Takenaka and Y. Nakano, "Multimode interference bistable laser diode," IEEE Photon. Technol. Lett., vol. 15, no. 8, pp. 1035-1037, Aug. 2003.

[4] R. Clavero, F. Ramos, J. Martinez, and J. Marti, "All-optical flip-flop based on a single SOA-MZI," IEEE Photon. Technol. Lett., vol. 17, no. 4, pp. 843-845, Apr. 2005.

[5] J. Zhou, C. Michael, and T. Makino, "All-optical bistable switching dynamics in $1.55 \mu \mathrm{m}$ two-segment strained multiquantum-well distributed-feedback lasers," J. Lightw. Technol., vol. 15, no. 2, pp. 342-355, Feb. 1997.

[6] M. Adams and R. Wyatt, "Optical bistability in distributed feedback semiconductor laser amplifiers," in Proc. Inst. Elect. Eng., Feb. 1987, vol. 134 , no. 1 , pp. $35-40$.

[7] D. Maywar, G. Agrawal, and Y. Nakano, "All-optical hysteresis control by means of cross-phase modulation in semiconductor optical amplifiers," J. Opt. Soc. Amer. B, vol. 18, no. 7, pp. 1003-1013, Jul. 2001.

[8] VPItransmissionmaker [Online]. Available: http://www.vpisystems.com 